\title{
Diffuse reflectance spectroscopy to monitor murine colorectal tumor progression and therapeutic response
}

\author{
Ariel I. Mundo \\ Gage. J. Greening \\ Michael J. Fahr, Jr. \\ Lawrence N. Hale \\ Elizabeth A. Bullard \\ Narasimhan Rajaram \\ Timothy J. Muldoon
}

Ariel I. Mundo, Gage. J. Greening, Michael J. Fahr Jr., Lawrence N. Hale, Elizabeth A. Bullard, Narasimhan Rajaram, Timothy J. Muldoon, "Diffuse reflectance spectroscopy to monitor murine colorectal tumor progression and therapeutic response," J. Biomed. Opt. 25(3), 035002 (2020), doi: $10.1117 / 1 . J B O .25 .3 .035002$ 


\title{
Diffuse reflectance spectroscopy to monitor murine colorectal tumor progression and therapeutic response
}

\author{
Ariel I. Mundo, ${ }^{a}$ Gage. J. Greening, ${ }^{\text {a }}$ Michael J. Fahr Jr., \\ Lawrence N. Hale, ${ }^{c}$ Elizabeth A. Bullard, ${ }^{a}$ Narasimhan Rajaram, ${ }^{a}$ \\ and Timothy J. Muldoon ${ }^{\mathrm{a}, *}$ \\ ${ }^{a}$ University of Arkansas, Department of Biomedical Engineering, Fayetteville, Arkansas, \\ United States \\ ${ }^{b}$ University of Arkansas, Department of Computer Science, Fayetteville, Arkansas, \\ United States \\ ${ }^{c}$ University of Arkansas, Department of Chemistry and Biochemistry, Fayetteville, \\ Arkansas, United States
}

\begin{abstract}
Significance: Many studies in colorectal cancer (CRC) use murine ectopic tumor models to determine response to treatment. However, these models do not replicate the tumor microenvironment of CRC. Physiological information of treatment response derived via diffuse reflectance spectroscopy (DRS) from murine primary CRC tumors provide a better understanding for the development of new drugs and dosing strategies in CRC.

Aim: Tumor response to chemotherapy in a primary CRC model was quantified via DRS to extract total hemoglobin content $(\mathrm{tHb})$, oxygen saturation $\left(\mathrm{StO}_{2}\right)$, oxyhemoglobin, and deoxyhemoglobin in tissue.

Approach: A multimodal DRS and imaging probe $(0.78 \mathrm{~mm}$ outside diameter) was designed and validated to acquire diffuse spectra longitudinally — via endoscopic guidance-in developing colon tumors under 5-fluoruracil (5-FU) maximum-tolerated (MTD) and metronomic regimens. A filtering algorithm was developed to compensate for positional uncertainty in DRS measurements

Results: A maximum increase in $\mathrm{StO}_{2}$ was observed in both MTD and metronomic chemotherapy-treated murine primary CRC tumors at week 4 of neoadjuvant chemotherapy, with $21 \pm$ $6 \%$ and $17 \pm 6 \%$ fold changes, respectively. No significant changes were observed in $\mathrm{tHb}$.

Conclusion: Our study demonstrates the feasibility of DRS to quantify response to treatment in primary CRC models.

(C) The Authors. Published by SPIE under a Creative Commons Attribution 4.0 Unported License. Distribution or reproduction of this work in whole or in part requires full attribution of the original publication, including its DOI. [DOI: 10.1117/1.JBO.25.3.035002]
\end{abstract}

Keywords: azoxymethane; colon cancer; diffuse reflectance spectroscopy; endoscopy; hemoglobin content; oxygen saturation; metronomic chemotherapy; neoadjuvant chemotherapy; phantom.

Paper 200003R received Jan. 10, 2020; accepted for publication Feb. 17, 2020; published online Mar. 6, 2020; corrected Mar. 27, 2020.

\section{Introduction}

Colorectal cancer (CRC) is the fourth leading cause of cancer death in the world, and in the United States, it accounted for 145,600 new cases in 2019. ${ }^{1,2}$ Preclinical research aimed at understanding the mechanisms of this disease and developing new therapies, frequently focuses on the use of ectopic xenografts or allografts in murine models to study molecular shifts in tumors. ${ }^{3-5}$ However, these xenograft/allograft animal models do not properly take into account the

*Address all correspondence to Timothy J. Muldoon, E-mail: tmuldoon@uark.edu 
influence of the tumor microenvironment ${ }^{6-8}$ and lack predictive power regarding clinical phase II performance, ${ }^{9}$ which limits the inferences from the data obtained from them. Primary mouse models of CRC, which more closely replicate primary tumors seen in humans-tumors that develop from dysplastic lesions within the colon epithelium itself-resemble the tumor microenvironment and anatomic location, making them better suited for the study of this disease. These models can be derived via carcinogen administration—such as azoxymethane (AOM) or in transgenic animals (such as APC ${ }^{\text {min }}$ ). ${ }^{10}$

In particular, these models can be used to study antitumor drugs and dosing strategies for neoadjuvant chemotherapy (NAC), which is administrated clinically before surgical resection with the aim of shrinking the tumor in patients with locally advanced disease (stage II or III). ${ }^{11}$ Typically, the standard NAC regimen is based on the maximum-tolerated dose (MTD) approach, ${ }^{8}$ which requires the cycling of treatment due to its associated toxicity and side effects (nausea, fever, pain). This cycling can have the unintended effect of allowing the tumors to recover in between administration of the antitumor agent. In contrast, the concept of low-dose continuous (metronomic) chemotherapy has been developed over the past 15 years, as it minimizes treatment side effects while targeting the endothelial cells of the tumor vasculature, which are not allowed to recover. ${ }^{12,13}$ This therapy has been explored as NAC in clinical studies of ovarian, cervical, and breast cancers, where tumor reduction was observed. ${ }^{14-16}$ Novel administration strategies for CRC - with the intent of understanding the mechanistic effects of this approach, as well as to identify potential clinically viable (endoscopic) biomarkers of positive therapy response-require the use of in vivo, primary models of the disease.

While primary CRC tumors may be removed from the mice following euthanasia or biopsied, longitudinal, in-vivo studies can track tumor progression (or involution) in response to therapy as well as interaction with the surrounding tissue. A variety of endoscopic imaging modalities that have been developed to study the colon of mice in vivo include optical coherence tomography (OCT), laser-induced fluorescence (LIF), label-free multiphoton microscopy (MPM), and Raman spectroscopy. ${ }^{17-19}$ These emerging imaging techniques have demonstrated that high-resolution imaging of the colon epithelium (OCT, MPM), extraction of fluorescence spectra from tissue (LIF), and spectral shifts to discriminate malignant tissue (Raman) are feasible in the preclinical setting but have not been used to track tumors longitudinally. However, these studies have highlighted the intrinsic challenge of in-vivo imaging and spectroscopy within the colonic environment, which complicates reproducibility and accuracy of measurements, making this an important factor that investigators need to consider for the acquisition of endoscopy-derived information in primary CRC models.

In this context, diffuse reflectance spectroscopy (DRS) is a maturing technology that features a minimally invasive, nonionizing optical technique that enables in-vivo quantification of tissue oxygen saturation $\left(\mathrm{StO}_{2}\right)$, total hemoglobin content $(\mathrm{tHb})$, oxyhemoglobin $\left(\mathrm{HbO}_{2}\right)$ and deoxyhemoglobin $(\mathrm{Hb})$. This technique has been applied in clinical studies of breast cancer to determine the response of tumors to $\mathrm{NAC}^{20,21}$ and to longitudinally monitor tumor response to treatment in subcutaneous xenografts. ${ }^{22}$ While DRS offers promising advantages for study of in-vivo tumor response to therapy, deployment via an endoscopic approach within murine models faces similar practical challenges as previously described.

Our group has previously used DRS in subcutaneous models of CRC, ${ }^{23}$ demonstrating the feasibility and advantages of extracting in-vivo physiological information. However, as the diameter of a mouse colon is below $4 \mathrm{~mm}$ and the thickness of the colonic wall ranges between 180 and $800 \mu \mathrm{m},{ }^{6,7}$ the miniaturization of DRS instrumentation presents different challenges: the probe needs to be miniaturized while maintaining an adequate signal-to-noise ratio, ${ }^{24}$ and peristalsis and subject breathing can both cause displacement between the probe and the tissue when data are collected, thereby introducing artifacts in the collected signal.

For a submillimeter diameter endoscopic DRS probe, the use of a pressure sensor to determine "optimal" contact with tissue is unlikely as miniature sensors are interferometry-based ${ }^{25}$ and they are not suited for direct contact with tissue. To overcome this limitation, we have devised an empirical analysis to model changes in DRS-obtained reflectance spectra due to motion or distance artifacts in order to filter the endoscopically acquired DRS data.

This study presents the design, validation, and endoscopic implementation of a smalldiameter $[0.78 \mathrm{~mm}$ outside diameter (OD)] multimodal optical imaging and DRS probe used 
in an AOM model of CRC to longitudinally quantify the response in vivo to different NAC (MTD or metronomic). To the best of our knowledge, this is the first study that compares endoscopically acquired DRS-derived physiological parameters from a model of CRC using different chemotherapy regimens.

\section{Materials and Methods}

\subsection{Multimodal Optical Imaging and Diffuse Reflectance Spectroscopy Probe Design}

A small diameter $(0.78 \mathrm{~mm}$ OD) multimodal optical imaging and DRS probe (Myriad Fiber Imaging, Dudley, Massachusetts) was designed to be deployed through the biopsy port of a commercial veterinary colonoscope (Karl Storz COLOView). The distal end of the probe comprises four silica optical fibers with a core diameter of $100 \pm 3 \mu \mathrm{m}$ each, individually surrounded by a polyimide buffer; and an imaging/DRS fiber (Fujikura FIGH-10-350S) with an image circle diameter of $325 \pm 20 \mu \mathrm{m}$, a fiber center-to-center distance of $3 \mu \mathrm{m}$, coated with silicon resin [Fig 1(b)]. The center-to-center distance between each individual optical fiber and the imaging fiber is $\sim 290 \mu \mathrm{m}$.

The total length of the probe is $2 \mathrm{~m}$. At the distal end, the fibers are bundled together under a stainless-steel sheath for the first $3 \mathrm{~mm}$ [Figs. 1(a) and 1(b)], after which they are under a black polyimide cover for $1 \mathrm{~m}$, finally branching individually for the remaining distance $(1 \mathrm{~m})$ each finishing in a SMA095 connector. Using a previously reported microendoscopy system ${ }^{26,27}$ the imaging component of the probe was characterized (Probe imaging characterization in the Supplementary Material).

\subsection{Probe Diffuse Reflectance Spectroscopy Characterization: Calibration, Validation, and Sampling Depth}

The inverse lookup table (LUT) approach was used for DRS, which has been described elsewhere. ${ }^{28-30}$ Briefly, liquid (calibration) phantoms with known optical properties $\left(\mu_{a}, \mu_{s}^{\prime}\right)$ were created to span a biological relevant range of $\mu^{\prime}{ }_{s}$ and $\mu_{a}$ in tissue. ${ }^{31,32}$ The phantom optical properties, system configuration, and LUT appear in Figs. S2(a), S2(b), S2(e), and S3(a) in the Supplementary Material. Detailed methods appear in DRS Calibration in the Supplementary Material.

For validation, five validation phantoms were created [Figs. S2(c) and S2(d) in the Supplementary Material] and a nonlinear optimization routine was used to extract the optical properties $\left(\mu_{a}\right.$ and $\left.\mu_{s}^{\prime}\right)$ using previously described constraining equations and boundary conditions $^{23,28}$ and Fig. S3(b) in the Supplementary Material. The methodology for this step appears in DRS Validation in the Supplementary Material.

Sampling depth of the DRS configuration of the multimodal probe was defined as the depth reached by $50 \%$ of the photons and calculated as reported elsewhere. ${ }^{23,33}$ Methods are described in detail in Sampling Depth in the Supplementary Material.

(a)

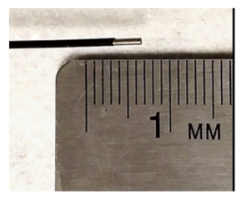

(b)

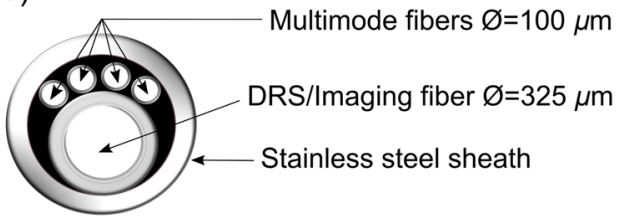

Fig. 1 Multimodal DRS probe. (a) Distal end of the probe showing the optical fibers $(\varnothing=100 \mu \mathrm{m}$ each, polyimide buffer for each fiber in gray) and the DRS/imaging fiber ( $\varnothing=325 \mu \mathrm{m}$, silicone resin coating in dark gray). (b) Optical and DRS/imaging fiber dimensions and configuration. 


\subsection{Animal Studies: Azoxymethane Model and Treatments}

An AOM model of CRC was used. Nine-week-old female A/J mice (000646 The Jackson Laboratory, Maine, $n=23$ ) received AOM injections (A5486, Millipore Sigma) at a dose of $10 \mathrm{mg} / \mathrm{kg}$ once a week for 6 weeks. All procedures were approved by the University of Arkansas Institutional Animal Care and Use Committee. Mice were acclimated for 1 week upon arrival, after which the active treatment phase began. During the AOM phase, weight was assessed daily, and subcutaneous sterile saline injections were performed if weight loss was $>10 \%$ when compared to the weight of the first day of injection.

Mice strain and AOM dosage were based on previously reported criteria and values. ${ }^{34}$ The animals were housed in a 12:12 h light-dark cycle facility and received rodent chow (8640, Teklad) and water ad libitum. Wet rodent chow was also provided to the mice during the active AOM treatment phase.

At 20 weeks post first AOM injection, the mice started the 6-week treatment phase. Mice were randomly distributed in MTD chemotherapy $(n=8)$, metronomic chemotherapy (MG, $n=8)$, and control (CG, $n=7$ ) treatments groups. Chemotherapy was administered 5-FU (Millipore Sigma, F6627). The MTD group received $20 \mathrm{mg} / \mathrm{kg}$ of 5-FU intraperitoneally (i.p.) every day for 5 days every other week, and the MG group received $8 \mathrm{mg} / \mathrm{kg}$ of 5-FU i.p. every other day three times a week. Control mice received $200 \mu \mathrm{L}$ of sterile saline i.p. every other day three times a week [Fig. 2]. MTD 5-FU dosage was based on the maximum dose reported by Azrak et al., ${ }^{35}$ and the break periods followed those seen in NAC in humans. MG dosage (70\% reduction) and therapy schedule were based on values reported previously. ${ }^{36,37}$

\subsection{Animal Studies: Endoscopic Procedures}

To perform integrated DRS and imaging on the endoscopic procedures, a commercial colonoscope unit (Karl Storz COLOView) was used, in a similar manner reported by other groups ${ }^{38,39}$ but in conjunction with the multimodal DRS probe. To achieve this, the DRS probe was deployed through one of the insufflation ports of the examination sheath [Fig. 3(a)] of the colonoscopy system, thus allowing visual determination of the position of the probe in the colon before acquiring data [Fig. 3(c)]. The configuration for data acquisition for the multimodal DRS probe was the same, as previously indicated in Sec. 2.2 and in DRS Calibration in the Supplementary Material. A footswitch-triggered shutter was implemented to block the light of the colonoscopy system for spectroscopic measurements [Fig. 3(b)]. Colonoscopy, spectroscopy, and combined displays were used to have a holistic view of the procedure.

At 18 weeks post first AOM injection, mice were anesthetized using $2 \%$ isoflurane and underwent exploratory imaging-only colonoscopies to assess tumor size. At 20 weeks, the tumors were determined to be roughly the diameter of the DRS probe (1 mm across), and treatment (5-FU or saline) was initiated. For the first week of treatment, colonoscopies where DRS

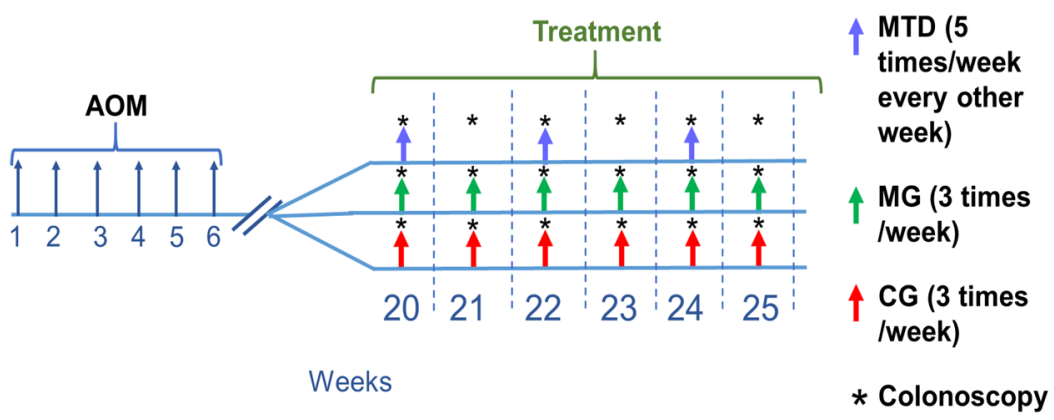

Fig. 2 Study timeline. All mice received AOM once a week for 6 weeks at a dose of $10 \mathrm{mg} / \mathrm{kg}$. At 20 weeks post first AOM injection, mice were divided in treatment groups (5-FU NAC or control). MTD mice received $5-\mathrm{FU}$ at a dose of $20 \mathrm{mg} / \mathrm{kg}$ (5 times/week every other week), MG mice received $8 \mathrm{mg} / \mathrm{kg}$ of 5-FU every other day (3 times/week), and CG mice received $200 \mu \mathrm{L}$ of sterile saline ( 3 times/week). Colonoscopies were performed once a week per mouse on the treatment phase, 1 day after the first injection (week 20) and every 7 days subsequently. 
(a)

(c)

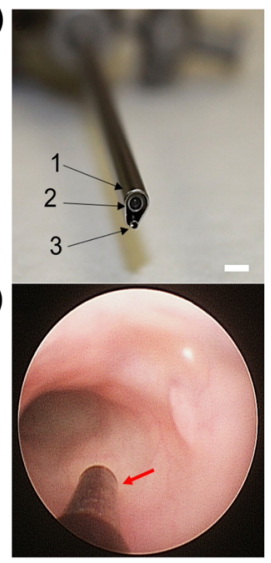

(b)

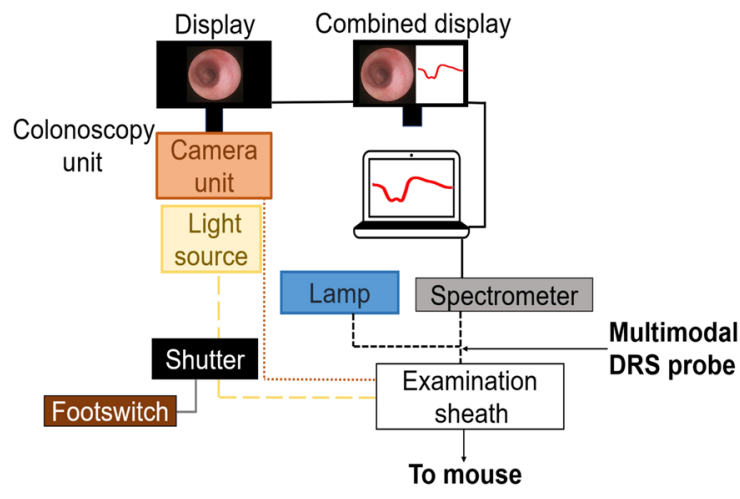

Fig. 3 Integrated colonoscopy system setup and in-vivo visualization. (a) System configuration used for colonoscopy/DRS acquisition: (1) examination sheath: (2) colonoscopy camera: (3) multimodal DRS probe. Scale bar: $2.3 \mathrm{~mm}$ (b) System setup for the colonoscopy/DRS data acquisition. The shutter is activated by a footswitch to block the light from the colonoscopy light source thus enabling only the light of the spectroscopy lamp for DRS measurements. (c) Visualization of the multimodal DRS probe within the colonoscopy camera FOV during the colonoscopy procedure.

data was collected were performed $24 \mathrm{~h}$ after the first injection, and subsequently repeated every 7 days for 6 weeks per mouse [Fig. 2].

Position of the probe with the tumor was determined visually by keeping the tumor within the camera field of view (FOV) and advancing the DRS probe until it touched the tumor. If deformation of the tumor was visible due to the contact of the probe, the procedure was repeated until contact of the probe caused no visible deformation on the tumor. At that point, the footswitch [Fig. 3(b)] was triggered, the light from the colonoscopy unit was blocked, and contact of the DRS probe on the tumor was confirmed if no light from the spectroscopy lamp was seen to be leaking into the environment. A minimum of 10 spectra were collected per tumor at an integration time of $70 \mathrm{~ms}$. Background (noise) and calibration data were collected, as previously described.

At the end of the 6-week period, mice underwent a final colonoscopy where the colon was stained with $1 \%$ fluorescein (F6377, Millipore Sigma) and microendoscopy images were acquired using the system configuration described in Fig. S1(b) in the Supplementary Material, and the deployment of the multimodal DRS probe as in Fig. 3(a) using 5 dB gain, 100 ms shutter at a rate of $10 \mathrm{fps}$ using the FlyCapture ${ }^{\circledR}$ software. After the colonoscopy/microendoscopy procedure, mice were euthanized under $4 \%$ isoflurane via cervical dislocation. Colons were excised, placed in OCT, and flash-frozen in liquid isopentane and stored at $-80^{\circ} \mathrm{C}$.

\subsection{Positional Sensitivity of the Multimodal Diffuse Reflectance Spectroscopy Probe}

Although great care was taken when the DRS data were acquired, the inherent moving of the colonic environment (due to peristalsis, mouse breathing, and operator movement) would introduce artifacts in the spectra, and therefore a threshold would be required to filter the data before quantifying physiological values. This issue was addressed in two steps: First, an ex-vivo analysis was used to develop an empirical model for the changes in reflectance due to variations in the positioning of the probe relative to the tissue. Later, the changes in reflectance obtained from the ex-vivo model were compared to the in-vivo data to determine a threshold in reflectance to reject spectra with angular or displacement artifacts.

\subsubsection{Ex-vivo analysis of the effects of probe contact angle and distance from tissue}

The ex-vivo analysis was performed to model the changes in reflectance due to positive displacement (when the DRS probe would be away from the tissue), angle of contact between the probe 
and the tissue, and negative displacement (when the probe deforms the tissue). Since the thickness of mouse colon is $<400 \mu \mathrm{m}$, the tissue cannot be placed directly over a surface to acquire DRS data as the specular reflection from the material-due to the close proximity of the light source-would cause contamination in the signal.

A system was devised [Fig. 4] where the tissue would be placed on top of a block of polydimethylsiloxane (PDMS) with thickness of $26 \mathrm{~mm}$ (PDMS block A) and mounted with staples. The block had cut out a $1 \times 1 \mathrm{~cm}$ section lengthwise, which had black metal foil surrounding it and extending $11 \mathrm{~mm}$ outside of the block (37 mm total length), thus creating a "photon well" that would ensure that no reflected light from the PDMS surface would contaminate the signal. The block was placed on top of a 50-mL beaker with a block made of PDMS and black India ink at the bottom (PDMS block B) that acted as a black body $\left(\mu_{a}>100 \mathrm{~cm}^{-1}\right)$ to absorb any remaining photons that would reach the bottom of the system. The beaker was then placed on top of a micrometer $z$-stage and the multimodal DRS probe was attached to a digital goniometer fixed to a metal post. In this manner, angle and distance could be precisely tuned.

Freshly resected colons from two mice containing one tumor each were excised from euthanized mice, cut lengthwise to expose the tumors and rinsed with cold phosphate-buffered saline. Tissue was then mounted on the system and the $z$-stage was moved up until contact between the probe and the tissue was assessed. From there, the $z$-stage was lowered in $100 \mu \mathrm{m}$ increments to $1000 \mu \mathrm{m}$ as this would correspond to a positive displacement of 1.3 probe diameters and would be a plausible upper limit for the distance analysis. The $0 \mathrm{deg}$, in contact measurements were defined as "optimal" as they would represent the values obtained when no pressure, angle, or distance artifacts are present in the spectra. Spectra were collected per position [Fig. 6(a)] with an integration time of $70 \mathrm{~ms}$ with calibration and dark noise spectra acquired accordingly. The process was repeated when the angle was varied at $0 \mathrm{deg}, 5 \mathrm{deg}, 15 \mathrm{deg}$, and $30 \mathrm{deg}$ [Fig. 4] to determine changes in reflectance due to changes in contact angle. Finally, negative displacement influence was assessed by placing the probe in contact with the tissue and lifting the $z$-stage in $100 \mu \mathrm{m}$ increments up to $700 \mu \mathrm{m}$. These measurements were made only with a 0 deg configuration on the system [Fig. 6(b)].

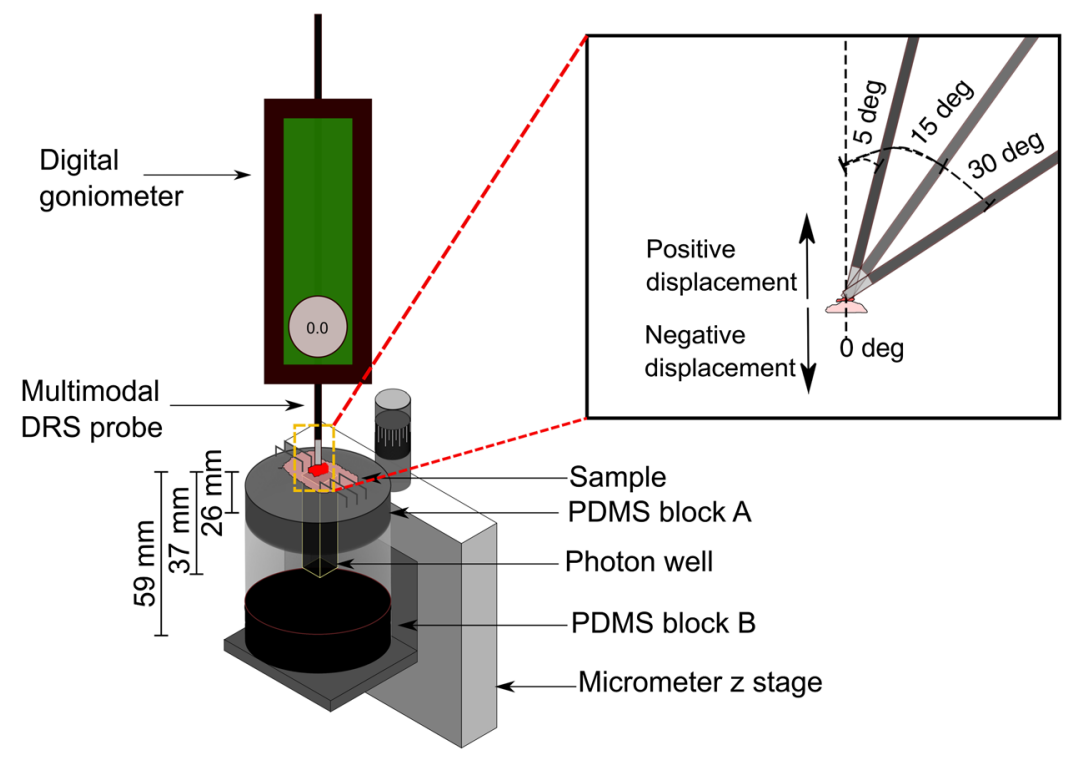

Fig. 4 Ex-vivo system setup for DRS analysis. Tissue was fixed using staples over a PDMS block that had a lengthwise $1 \times 1 \mathrm{~cm}$ section cut and surrounded by black metal foil thus forming a photon well. A micrometer $z$-stage was used to measure changes in distance in $100 \mu \mathrm{m}$ increments. Insert presents the variation of the angle of contact between the probe and the tissue for the 0 deg, $5 \mathrm{deg}, 15 \mathrm{deg}$, and $30 \mathrm{deg}$ measurements and the direction of positive and negative displacements between the probe and tissue. 


\subsubsection{In-vivo analysis of optimized probe position}

The ex-vivo reflectance from colonic tissue was compared to the in-vivo reflectance of tumors [Figs. 7(a)-7(c)] where optimal contact was assessed at the moment of spectral acquisition (tumor not deformed by negative displacement, gentle contact, no visible angular separation between the probe) for eight mice (four MTD and four CG). Each mouse had a minimum of five colonoscopies performed, therefore allowing the assessment of temporal changes (longitudinal data) to compare the behavior of in-vivo and ex-vivo reflectance to determine a threshold to discard data that had pressure, angle, or distance artifacts.

\subsection{Diffuse Reflectance Spectroscopy Postprocessing: Inverse Lookup Table Model}

The filtered in-vivo data were used to quantify optical properties and physiological values from tissue $\left(\mathrm{StO}_{2}, \mathrm{tHb}, \mathrm{HbO}_{2}\right.$, and $\left.\mathrm{Hb}\right)$ using the inverse LUT model between 475 and $685 \mathrm{~nm}$ using constraining equations and boundary conditions as reported elsewhere. ${ }^{23,28}$ Pigment packaging correction was not introduced in the absorption coefficient as the analysis was performed $>400 \mathrm{~nm} \cdot{ }^{29}$ Detailed methodology is presented in DRS post-processing (Inverse LUT model) in the Supplementary Material.

\subsection{Statistical Analysis}

The final physiological DRS-derived data were unbalanced with skewness in the distribution of the values (data not shown). The typical repeated measures analysis of variance (rmANOVA) could not be used to analyze the data, as this methodology requires complete observations per subject in order to incorporate it into the analysis and also assumes sphericity for the correlation of the repeated measurements, a condition that may not hold true. ${ }^{40}$

Therefore, a generalized least squares (GLS) method was used to assess the interaction of treatment and time on the DRS physiological data $\left(\mathrm{StO}_{2}, \mathrm{tHb}, \mathrm{HbO}_{2}\right.$, and $\left.\mathrm{Hb}\right)$, as this methodology models the correlations between observations in a more flexible way than the rmANOVA model and also supports the use of unbalanced data. ${ }^{41}$ The covariance structure was estimated using an autoregressive process of unequal variances for the observations across time, which was determined to be optimal in base of the log-likelihood value and examination of the correlation of measurements over time. A model where the effect of treatment, time, and their interaction could be assessed over the mean DRS-derived physiological data $\left(\mathrm{StO}_{2}, \mathrm{tHb}, \mathrm{HbO}_{2}\right.$, and $\mathrm{Hb}$ ) was constructed. The significance of fixed effects was set at $p<0.05$. Statistical analyses and data visualization were performed in $\mathrm{R}$ (The R Foundation, version 3.5.2), ${ }^{42}$ using the nlme and ggplot2 packages. $^{43,44}$

\section{Results}

\subsection{Validation of the Lookup Table-Inverse Model}

The percentage errors between the phantom optical properties extracted with the inverse LUT model and the known optical properties were quantified as a validation step [Fig. 2(b)]. Average errors were 9.4 and 6.7 for $\mu^{\prime}{ }_{s}$ and $\mu_{a}$, respectively [Figs. 5(a) and 5(b)].

\subsection{Ex-Vivo Changes in Reflectance: Variations Due to Positive or Negative Displacement}

The ex-vivo analysis revealed a minimal increase in reflectance for the first $300 \mu \mathrm{m}$ of positive displacement, reaching a maximum at a distance of $500 \mu \mathrm{m}$ and decreasing again in the 600 to $1000 \mu \mathrm{m}$ [Fig. 6(a)].

For negative displacement, spectra transitioned in the 500 to $600 \mathrm{~nm}$ range from a predominantly $\mathrm{HbO}_{2}$ spectra to an $\mathrm{Hb}$ spectrum [Fig. 6(b)]. The complete set of spectra appears in Figs. S5(a) and S5(b) in the Supplementary Material. 

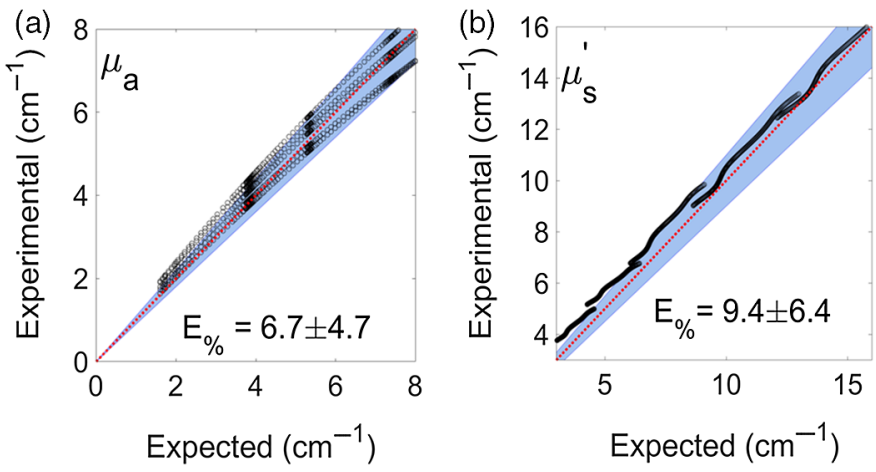

Fig. 5 (a) and (b) Percentage errors between the inverse LUT model calculated and the known optical properties of the validation phantoms. Both $\mu_{a}$ and $\mu^{\prime}{ }_{s}$ were below $10 \%$. Red line represents perfect agreement between the measures, black dots represent calculated optical properties per phantom, and blue background represents a $10 \%$ error margin.
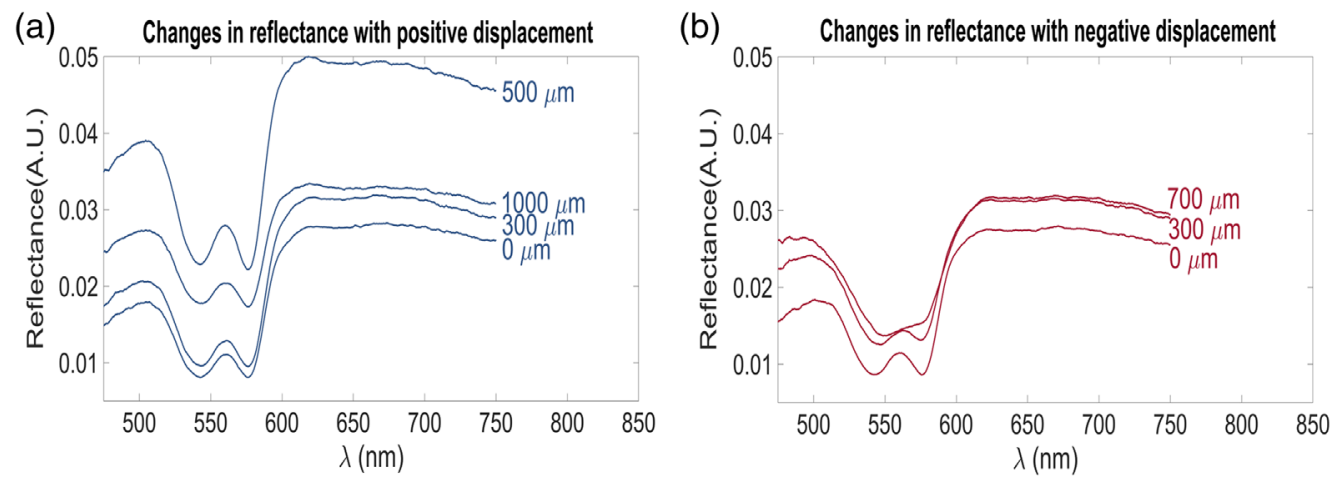

Fig. 6 (a) Representative changes in reflectance with positive displacement between the probe and the tissue. Labels per spectra correspond to the distance between the probe and the tissue according to the micrometer scale. (b) Representative changes in reflectance due to negative displacement between the probe and the tissue. Labels per spectra correspond to the distance advanced by the probe over the tissue, as recorded by the micrometer scale.

\subsection{Ex-Vivo and In-Vivo Optimal Reflectance: In-Vivo Reflectance Is Stable and Correlates with Reflectance Acquired Ex Vivo}

Measurements obtained in vivo demonstrated that reflectance $(R)$ remained repeatedly below 0.05 at $475 \mathrm{~nm}$ [Figs. 7(a) and 7(b)], in line with the values from the optimally acquired exvivo DRS data [Fig. 7(c)]. The Q bands of hemoglobin would be clearly defined and the region above $600 \mathrm{~nm}$ would have a similar linear profile.

\section{4 $\mathrm{Q}_{\text {ratio }}$ as a Metric to Filter Endoscopically Acquired Diffuse Reflectance Spectroscopy Data}

The results from Secs. 3.2 and 3.3 were used to calculate a ratio between the scattering region $\left(\lambda_{S}=630 \mathrm{~nm}\right)$ and the second $\mathrm{Q}$ band of hemoglobin $\left(\lambda_{Q}=575 \mathrm{~nm}\right)$ per each optimal ex-vivo spectra, defined as

$$
Q_{\text {ratio }}(i)=\left[\frac{R_{\lambda s(i)}}{R_{\lambda Q(i)}}\right]
$$

where $i$ denotes each recorded reflectance in the 475 to $685 \mathrm{~nm}$ range and $R_{\lambda s(i)}, R_{\lambda Q(i)}$ are the values of the $i$ ' th reflectance at 630 and $575 \mathrm{~nm}$, respectively. The $Q_{\text {ratio }}$ was calculated for all the ex-vivo spectra with different displacement/angular variations, which demonstrated that changes 


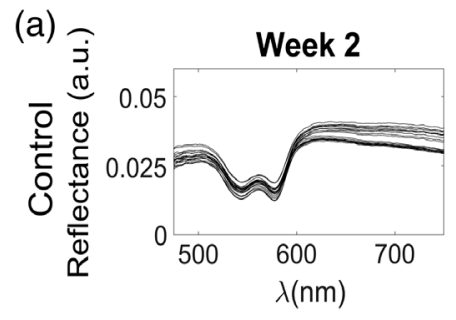

(b)

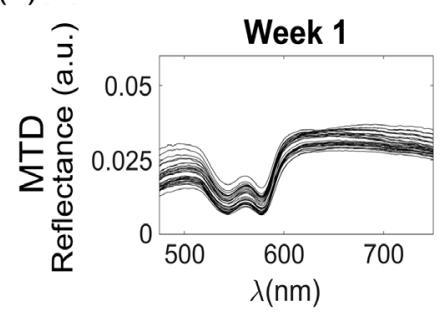

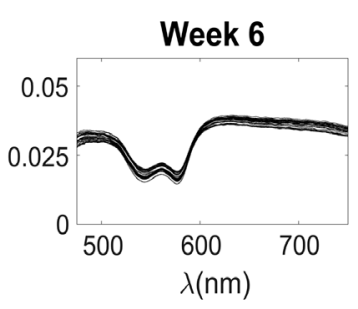

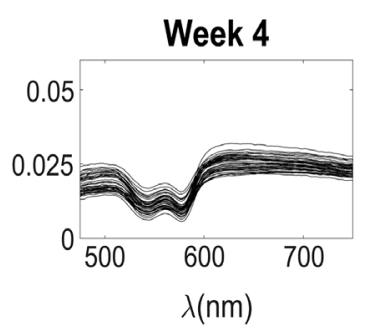

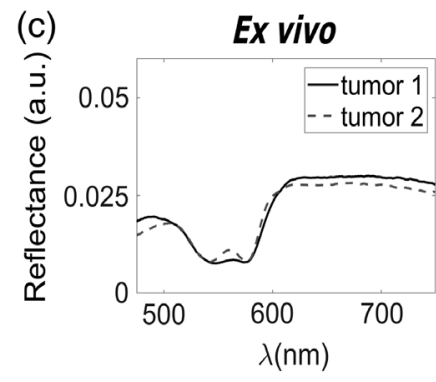

(d)

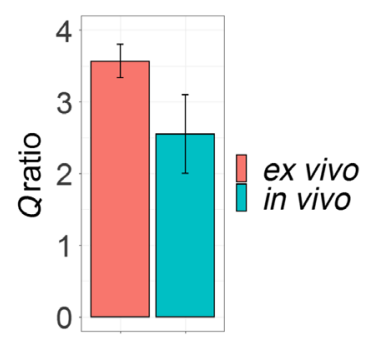

Fig. 7 Representative optimal longitudinal reflectance and $Q_{\text {ratio }}$ values. (a) In-vivo optimal reflectance-defined as tumor not deformed by negative displacement, gentle contact, no visible angular separation between the probe-acquired for the first day of the indicated week for weeks 2 and 6 for CG mouse. (b) In-vivo optimal reflectance for weeks 1, and 4 for treated (MTD) mouse. (c) Exvivo reflectance from tumors from CG (tumor 1) and MG (tumor 2) mice. Behavior of the spectra was longitudinally consistent in vivo and with the ex vivo reflectance, thus providing support to the use of a ratio to filter outliers. (d) $Q_{\text {ratio }}$ calculated for optimally acquired data in vivo (gentle contact and no separation visible) and ex vivo ( 0 deg, in contact). Bars are means; error bars represent $\pm 1 \mathrm{SD}$.

in angle/displacement resulted in different $Q_{\text {ratio }}$ s, whereas values for the 0 deg and 0 to $300 \mu \mathrm{m}$ positive displacement configuration were similar in both tumors [Fig. S6 in the Supplementary Material]. The $Q_{\text {ratio }}$ for 25 ex-vivo optimal spectra were calculated and demonstrated a mean of $3.56 \pm 0.23$, whereas the $Q_{\text {ratio }}$ (from 258 spectra) was calculated from in-vivo data, with a mean value $2.55 \pm 0.55$ [Fig. 7(d)]. The similarity between the ex-vivo and in-vivo $Q_{\text {ratio }}$ s supported the use of this metric to differentiate optimal data from data with displacement or angle issues.

Therefore, a filtering algorithm was constructed to discard data that had angular or displacement (positive or negative) artifacts to avoid biasing the DRS data to be analyzed. Detailed description of the algorithm and the corresponding flowchart are summarized in the Filtering Algorithm and Fig. S6 in the Supplementary Material].

\subsection{Treatment Outcomes: Endoscopic and Microendoscopic Comparisons}

Tumors were endoscopically monitored over time during the treatment (chemotherapy or saline) phase [Fig. 2]. Tumor regression was observed in the majority of the MTD-treated tumors between the treatment and nontreatment weeks [Fig. 8(d)]. No tumor regression was observed in the MG-treated tumors and CG tumors [Figs. 8(e) and 8(f)]. Vasculature was visible on the surface of all tumors.

\subsection{Diffuse Reflectance Spectroscopy-Derived Physiological Parameters from Tumors}

Using the inverse LUT model, physiological parameters $\left(\mathrm{StO}_{2}, \mathrm{tHb}, \mathrm{HbO}_{2}\right.$, and $\left.\mathrm{Hb}\right)$ were quantified from the AOM-induced tumors. For $\mathrm{StO}_{2}$, longitudinal mean values ranged between $66 \%$ to $77 \%$ (CG), $63 \%$ to $76 \%$ (MG) and $71 \%$ to $86 \%$ (MTD) [Fig. 9(a)]. Mean $\mathrm{StO}_{2}$ increase was observed in the NAC groups, where the maximum fold change was observed at week 4 (17\% for MG and 21\% for MTD) [Fig. 9(e)]. 
(a)

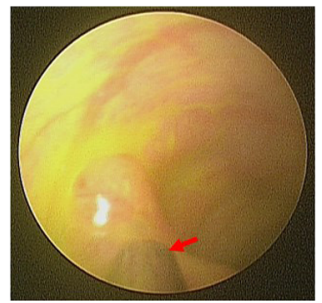

(b)

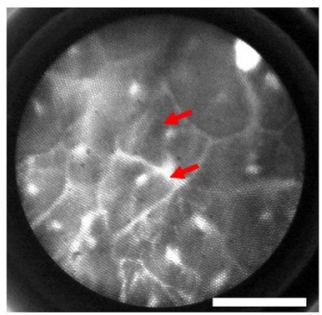

(c)

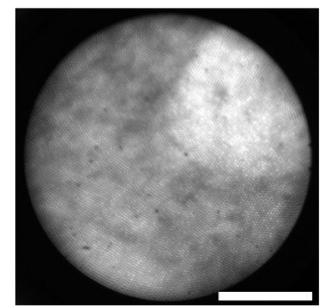

(d)

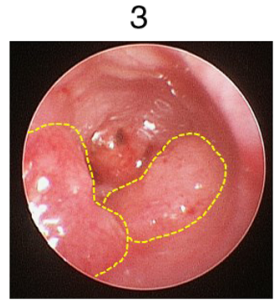

(e)

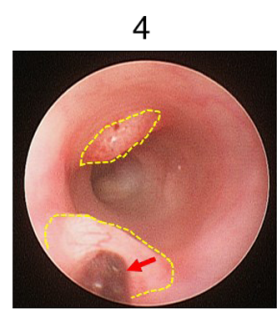

(f)

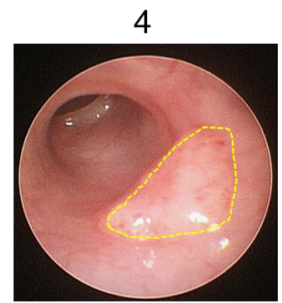

Week
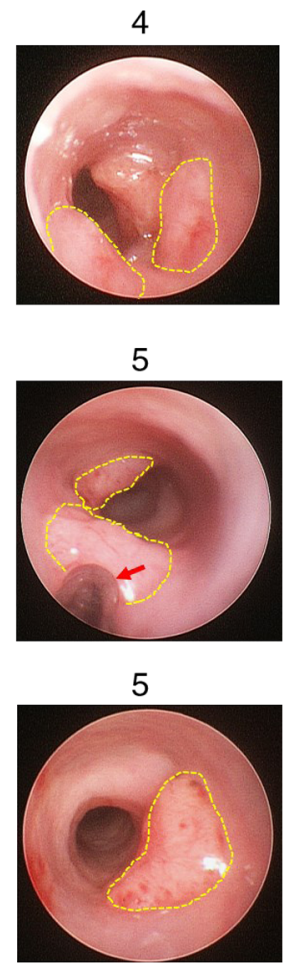

Fig. 8 Representative longitudinal endoscopic and microendoscopic imaging of AOM-induced tumors. (a) Endoscopic image of fluorescein stain in vivo, where red arrow indicates the multimodal DRS probe. (b) Normal colon imaged using the microendoscopy configuration. Arrows indicate colonic crypts. Scale bar: $100 \mu \mathrm{m}$. (c) AOM-induced tumor imaged using the microendoscopy configuration. Scale bar $100 \mu \mathrm{m}$. (d) Representative tumors of the MTD group. Regression of the tumor is visible between weeks 3 and 4. (e) Representative tumors of the MG group. (f) Representative tumors of the CG group. In (e) and (f) the size of the tumor increases over time. Tumor vasculature is readily visible in the tumors across all groups. All pictures have been enhanced to emphasize tumor and colonic morphology, yellow dashed lines in (d)-(f) outline the visible volume of the tumor, red arrows indicate the multimodal DRS probe.

For $\mathrm{HbO}_{2}$, mean values ranged between 2.7 to $3.7 \mathrm{mg} / \mathrm{mL}$ (CG), 2.8 to $3.6 \mathrm{mg} / \mathrm{mL}$ (MG), and 3.4 to $4.1 \mathrm{mg} / \mathrm{mL}$ (MTD) [Fig. 9(b)]. The maximum fold change was 20\% (MTD and MG) at weeks 4 and 5, respectively [Fig. 9(f)]. For $\mathrm{Hb}$, mean values ranged between 1.0 to $1.5 \mathrm{mg} / \mathrm{mL}$ (CG), 0.9 to $1.7 \mathrm{mg} / \mathrm{mL}$ (MG), and 0.6 to $1.4 \mathrm{mg} / \mathrm{mL}$ (MTD) [Fig. 9(c)]. A decreasing trend of $\mathrm{Hb}$ was observed in the MTD and MG groups, reaching a $40 \%$ and $54 \%$ reduction at week 4 [Fig. $9(\mathrm{~g})]$.

Across all groups, tHb values ranged between 4.1 to $5.0 \mathrm{mg} / \mathrm{mL}$ (CG), 4.3 to $5.0 \mathrm{mg} / \mathrm{mL}$ (MG), and 4.3 to $4.8 \mathrm{mg} / \mathrm{mL}$ (MTD) [Fig. 9(d)]. No specific trend in the change of tHb was observed [Fig. 9(h)].

For $\mathrm{StO}_{2}$, the interaction of chemotherapy (MTD or MG) and the second-degree polynomial term of time was significant ( $p=0.0071$ and $p=0.049$, respectively). For $\mathrm{Hb}$, interaction of MG and the second-degree polynomial term was significant $(p=0.007)$. In the case of $\mathrm{HbO}_{2}$, interactions between chemotherapy (MTD or MG) and the second-degree polynomial term were significant ( $p=0.0457$ and $p=0.036$, respectively). For tHb, the interaction of MG and the fifth-degree polynomial term was significant $(p=0.0078)$.

\section{Discussion}

Tumor response to therapy is an important step that needs to be addressed in the development of new drugs, dosing schemes, and treatment strategies that can be translated to the clinic. In this 


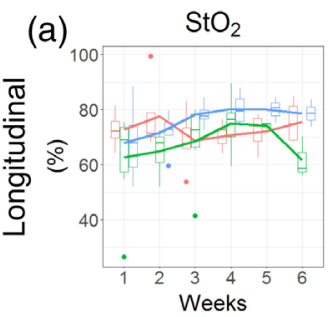

(e)

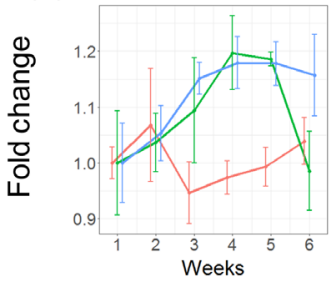

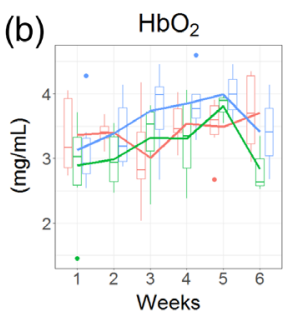

(f)

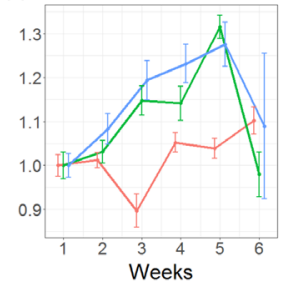

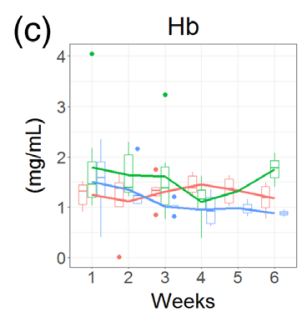

(g)

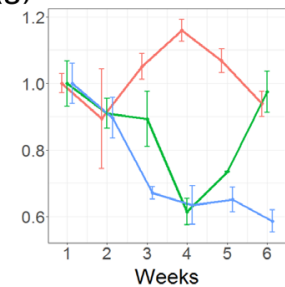

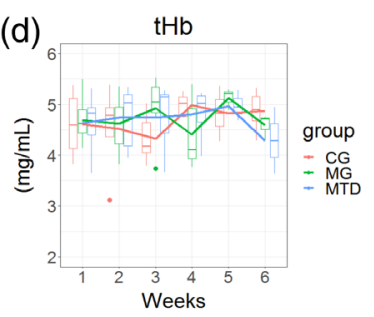

(h)

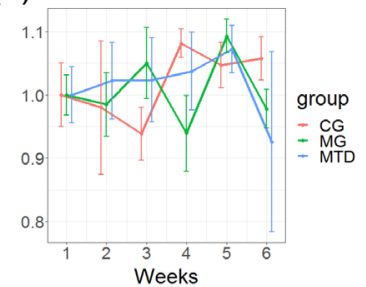

Fig. 9 Longitudinal physiological DRS-derived values for tumors under MTD ( $n=3$ to 8 ), MG ( $n=2$ to 8 ), and CG ( $n=3$ to 7) groups. (a)-(d) Longitudinal changes in $\mathrm{StO}_{2}, \mathrm{HbO}_{2}, \mathrm{Hb}$, and $\mathrm{tHb}$ for all groups. Each line represents the physiological data extracted using the inverse LUT model per group per day; boxplots represent the Q1 to Q3 interquartile range per group per day; central line of boxplot is the median value. Points represent outliers. (e)-(h) Longitudinal fold changes in $\mathrm{StO}_{2}, \mathrm{HbO}_{2}, \mathrm{Hb}$, and $\mathrm{tHb}$ compared to the value from week 1 for each group; error bars represent $\pm \mathrm{SE}$ /baseline value. Longitudinal trends per mouse and group for $\mathrm{StO}_{2}$ and $\mathrm{tHb}$ appear in Fig. S8 in Supplementary Material.

study, DRS was used to extract physiological data from tumors responding to therapy administration in vivo, and the inverse LUT model was used due to the scale of the system. ${ }^{45}$ Mean percentage errors for $\mu^{\prime}$ and $\mu_{a}$ were $9.4 \%$ and 6.7\%, respectively [Figs. 5(a) and 5(b)], in line with values reported in the literature. ${ }^{46-48}$ The sampling depth for DRS was $263 \pm 7 \mu \mathrm{m}$ at $542 \mathrm{~nm}$.

Diffuse spectra were acquired in developing colon tumors treated with 5-FU using MTD and metronomic regimens. However, as the previously mentioned endoscopic imaging studies have highlighted, variability in the endoscopically acquired signal due to distance from the tissue surface (positive or negative) and angular position between the probe and the tissue are factors that need to be properly controlled in order to accurately quantify tissue optical properties (and extracted physiological data). The orthogonal subtraction method is a statistical approach that accounts for this type of artifact introduced in the signal, but its application in this context is limited by the amount of data required to construct the orthogonal matrix, and because the assumptions required for this methodology may not hold true due to the scale of the system used in this study. ${ }^{49}$

Changes in reflectance due to positional probe variations have been analyzed previously, but the results from these studies are mixed, ranging from increase in reflectance due to pressure ${ }^{50}$ to a decrease in the signal due to the same reason. ${ }^{51}$ Also, datasets are sometimes incomplete, as indicated by Cugmas et al., ${ }^{52}$ thereby complicating inferences from those studies that could be applicable to our system. Therefore, an empirical model based on the fact that the optimally acquired $Q_{\text {ratio }}$ ex vivo $(3.56 \pm 0.23)$ was similar to the in vivo observed value for optimally acquired spectra $(2.55 \pm 0.55)$, [Fig. 7(d)] served as the basis for the use of this metric within an algorithm to automatically filter the data obtained by our submillimeter DRS probe. This methodology allowed us to obtain results specific to our system geometry and tissue of interest. Furthermore, the previously reported use of ratios in reflectance to discriminate between tissues and grade malignancies in the oral mucosa ${ }^{53,54}$ provided support to the rationale used to filter representative spectra and data with angular or distance artifacts.

In this study, we hypothesize that the observed variations in reflectance due to positive displacement of the probe [Figs. 6(a) and S5(a) in the Supplementary Material] can be explained by the liquid meniscus between the tip of the probe and the tissue for the first $300 \mu \mathrm{m}$, which stabilizes the signal. When the liquid meniscus and the tip of the probe separate (distance 
$>300 \mu \mathrm{m}$ ), a sharp increase is observed due to specular reflection caused by the surface of the liquid layer. Finally, as the probe moves farther away from tissue, the reflectance decreases as less light reaches the collecting fiber. During negative displacement, reflectance increased, but on a smaller scale when compared to the previously mentioned positive distance-induced changes. However, in the 500- to 600-nm range- the region where hemoglobin absorption occurs-an increased flattening of the $\mathrm{Q}$ bands was seen with incremental negative distance, a behavior previously reported by Popov et al. ${ }^{55}$ and $\mathrm{Ti}$ and Lin,${ }^{56}$ which is attributed to blood displacement and deoxygenation.

For the DRS-extracted physiological values, the observed $\mathrm{StO}_{2}$ during the first week of measurements ranged between $65 \%$ and $76 \%$ across all groups [Fig. 9(a)], in contrast with values extracted from subcutaneous models, where values below $50 \%$ have been reported. ${ }^{23,57} \mathrm{We}$ hypothesize that the observed difference can be partially explained by the size of the malignancy, as primary CRC tumors are much smaller (diameter $\sim 1 \mathrm{~mm}$ ) than their subcutaneous counterparts, which accordingly would be more hypoxic due to changes in perfusion and necrosis as a result to tumor growth. ${ }^{58}$ But it is also possible that higher $\mathrm{StO}_{2}$ values are caused by the blood present on the readily visible superficial vasculature in all groups [Fig. 8], which can be quantified in future studies to address this possibility.

In the NAC groups (MTD or metronomic), an increasing pattern over time was observed in both groups for $\mathrm{StO}_{2}$ and $\mathrm{HbO}_{2}$ [Figs. 9(a) and 9(b)], coupled with a decrease in $\mathrm{Hb}$ and no trend for tHb [Figs. 9(c) and 9(d)]. Chemotherapy (MTD or metronomic) and its interaction with the second-degree polynomial term were significant for $\mathrm{StO}_{2}$ and $\mathrm{HbO}_{2}(p=0.0071$ and $p=0.049$ for $\mathrm{StO}_{2}, p=0.0457$ and $p=0.036$ for $\mathrm{HbO}_{2}$, respectively), which indicate that the trends do not follow a linear pattern. Finally, the interaction of MG and the second-degree polynomial term was significant for $\mathrm{Hb}(p=0.007)$. While the sample size presents a limitation in this study, it is interesting to note that both NAC treatments cause similar changes in $\mathrm{StO}_{2}, \mathrm{HbO}_{2}$, and $\mathrm{Hb}$ over time. Based on our findings, we speculate that the observed changes are possibly caused by a reduced oxygen demand due to necrosis, which would explain the reported increase in $\mathrm{StO}_{2}$ and $\mathrm{HbO}_{2}$, whereas tHb remained constant. This question will be formally addressed in future studies.

Moreover, our results are in line with previously reported longitudinal trends in the change of $\mathrm{StO}_{2}$ and $\mathrm{HbO}_{2}$ due to MTD chemotherapy in subcutaneous models. ${ }^{22,59}$ For the metronomic regimen, the lack of a trend for $\mathrm{tHb}$ (indicating no significant treatment effect) and the increase in $\mathrm{StO}_{2}$ due to treatment suggest that the treatment did not affect the vascular network of the tumors, as suggested by Fioravanti et al. ${ }^{36}$ In contrast to the conclusions of that study, our results suggest that a 70\% reduction on the MTD dose of 5-FU is still capable of producing similar physiological changes $\left(\mathrm{StO}_{2}, \mathrm{HbO}_{2}\right.$, and $\left.\mathrm{Hb}\right)$ as in the MTD regimen due to the fold values observed, although it is not capable of producing tumor regression, which was observed visually (endoscopically) in the MTD regimen [Figs. 8(d) and 8(e)]. The GLS approach ${ }^{41}$ was chosen since the data acquired were unbalanced, and the sample size limits the assumption of a spherical variance-covariance matrix (as required in rmANOVA). ${ }^{40}$ Moreover, the GLS method was preferred as a linear-mixed effects model approach showed that a random effect of subject variability over time was not significant (data not shown).

Our results demonstrate the feasibility and associated challenges in the use of DRS to track the response to treatment in a primary CRC mouse model. To the best of our knowledge, there are limited longitudinal preclinical studies for NAC in this context that monitor tumor hemodynamics. This is the first study that compares physiological information from endoscopically acquired longitudinal DRS data from different NAC strategies. Owing to the ongoing interest in metronomic NAC and the possibility of using this type of therapy as an alternative as NAC in $\mathrm{CRC}$, this study serves as an initial step toward the refining and characterization of this therapeutic approach.

Limitations to the present study include the sample size per group, which limits the size effect observed between treatment groups, and the size of the tumors in the model used $(\sim 1 \mathrm{~mm}$ diameter), which are smaller than their human counterparts $(\sim 44 \mathrm{~mm} \text { diameter })^{60}$ as the microenvironment landscape is expected to be different, particularly in regard to oxygenation. Also, the model selected is chemically induced and presents different metabolic activity when compared to human tumors. ${ }^{61}$ Finally, the selected metronomic dosage and schedule is also a 
limitation as changes in the frequency of the treatment or fraction of reduction of the dose can potentially correlate with different physiological values.

\section{Conclusion}

This study outlines the calibration, validation, and implementation of a multimodal DRS probe in a primary CRC murine model with different NAC regimens. Optical errors for the DRS system were comparable to those reported in the literature. In-vivo DRS-derived physiological values were computed longitudinally for each group, where NAC (metronomic or maximum-tolerated) caused an increase in $\mathrm{StO}_{2}$ and $\mathrm{HbO}_{2}$ and a decrease in $\mathrm{Hb}$ in tumors, whereas no significant changes in $\mathrm{tHb}$ were observed. These results provide an initial step in understanding and quantifying tumor response in different chemotherapeutic regimens. The developed system enables the acquisition of in-vivo DRS information in a primary CRC murine model, which could potentially be used to determine tumor response to treatment and guide the development of new drugs for CRC. The concept of a deployable DRS probe could also be possibly translated to the study of other types of cancer that are diagnosed and studied via endoscopy, such as biliary, esophageal, or stomach cancer.

\section{Disclosures}

The authors declare no conflicts of interest related to this article.

\section{Acknowledgments}

This work was supported by the National Science Foundation CAREER Award (CBET 1751554), the National Cancer Institute (1R15 CA202662), the Arkansas Biosciences Institute, and the Fulbright Faculty Development Award (A. I. M.). A.I. M. thanks Sina Dadgar for comments on diffuse reflectance spectroscopy postprocessing and system setup.

\section{References}

1. National Cancer Institute, Cancer of the Colon and Rectum-Cancer Stat Facts, https://seer .cancer.gov/statfacts/html/colorect.html (2019).

2. M. Arnold et al., "Global patterns and trends in colorectal cancer incidence and mortality," Gut 66(4), 683-691 (2017).

3. M. L. Reyzer et al., "Early changes in protein expression detected by mass spectrometry predict tumor response to molecular therapeutics," Cancer Res. 64(24), 9093-9100 (2004).

4. K. K. Frese and D. A. Tuveson, "Maximizing mouse cancer models," Nat. Rev. Cancer 7(9), 645-658 (2007).

5. M. M. Taketo and W. Edelmann, "Mouse models of colon cancer," Gastroenterology 136(3), 780-798 (2009).

6. A. P. De Souza et al., "The role of selenium in intestinal motility and morphology in a murine model of Trypanosoma cruzi infection," Parasitol. Res. 106(6), 1293-1298 (2010).

7. N. Deshpande et al., "Quantification and monitoring of inflammation in murine inflammatory bowel disease with targeted contrast-enhanced US," Radiology 262(1), 172-180 (2012).

8. C. J. Allegra et al., "Phase III trial assessing bevacizumab in stages II and III carcinoma of the colon: results of NSABP protocol C-08," J. Clin. Oncol. 29(1), 11-16 (2011).

9. T. Voskoglou-Nomikos, J. L. Pater, and L. Seymour, "Clinical predictive value of the in vitro cell line, human xenograft, and mouse allograft preclinical cancer models," Clin. Cancer Res. 9(11), 4227-4239 (2003).

10. A. R. Moser et al., "APC ${ }^{\mathrm{Min}}$ - a mouse model for intestinal and mammary tumorigenesis," Eur. J. Cancer 31A(7-8), 1061-1064 (1995). 
11. P. F. Engstrom et al., "Colon cancer," J. Natl. Compr. Cancer Network 7(8), 778-831 (2009).

12. R. S. Kerbel and B. A. Kamen, "The anti-angiogenic basis of metronomic chemotherapy," Nat. Rev. Cancer 4(6), 423-436 (2004).

13. D. Genfors and M. Albertsson, "Low-dose metronomic capecitabine (Xeloda) for treatment of metastatic gastrointestinal cancer: a clinical study," Arch. Cancer Res. 4 (2016).

14. N. Masuda et al., "A phase II study of metronomic paclitaxel/cyclophosphamide/capecitabine followed by 5-fluorouracil/epirubicin/cyclophosphamide as preoperative chemotherapy for triple-negative or low hormone receptor expressing/HER2-negative primary breast cancer," Cancer Chemother. Pharmacol. 74(2), 229-238 (2014).

15. J. R. Hildebrand et al., "Neoadjuvant metronomic chemotherapy in triple negative breast cancer (TNBC) (NCT00542191): updated results from a phase II trial," J. Clin. Oncol. 34 (2016).

16. Y. Aoki et al., "Neoadjuvant chemotherapy using low-dose consecutive intraarterial infusions of cisplatin combined with 5-fluorouracil for locally advanced cervical adenocarcinoma," Gynecol. Oncol. 81(3), 496-499 (2001).

17. L. P. Hariri et al., "Endoscopic optical coherence tomography and laser-induced fluorescence spectroscopy in a murine colon cancer model," Lasers Surg. Med. 38(4), 305-313 (2006).

18. A. Dilipkumar et al., "Label-free multiphoton endomicroscopy for minimally invasive in vivo imaging," Adv. Sci. 6(8), 1801735 (2019).

19. A. Taketani et al., "Raman endoscopy for the in situ investigation of advancing colorectal tumors in live model mice," Analyst 138(14), 4183-4190 (2013).

20. J. M. Cochran et al., "Tissue oxygen saturation predicts response to breast cancer neoadjuvant chemotherapy within 10 days of treatment," J. Biomed. Opt. 24(2), 11 (2019).

21. B. J. Tromberg et al., "Predicting responses to neoadjuvant chemotherapy in breast cancer: ACRIN 6691 trial of diffuse optical spectroscopic imaging," Cancer Res. 76(20), 5933-5944 (2016).

22. K. Vishwanath et al., "Using optical spectroscopy to longitudinally monitor physiological changes within solid tumors," Neoplasia 11(9), 889-900 (2009).

23. G. Greening et al., "Sampling depth of a diffuse reflectance spectroscopy probe for in-vivo physiological quantification of murine subcutaneous tumor allografts," J. Biomed. Opt. 23(8), 085006 (2018).

24. A. I. Mundo, G. J. Greening, and T. J. Muldoon, "Characterization of a multimodal endoscopically deployable veterinary spectroscopy and imaging probe to determine therapeutic response in a murine orthotopic tumor model," Proc. SPIE 10890, 108901L (2019).

25. M. Li, M. Wang, and H. P. Li, "Optical MEMS pressure sensor based on Fabry-Perot interferometry," Opt. Express 14(4), 1497-1504 (2006).

26. S. P. Prieto et al., "Fiber bundle microendoscopy for characterization of dysplastic lesions in colonic epithelium," in Biomed. Opt. 2014, p. BT3A.14 (2014).

27. S. P. Prieto et al., "Qualitative and quantitative comparison of colonic microendoscopy image features to histopathology," Proc SPIE 9328, 93280B (2015).

28. N. Rajaram, T. Nguyen, and J. W. Tunnell, "Lookup table-based inverse model for determining optical properties of turbid media," J. Biomed. Opt. 13(5), 050501 (2008).

29. N. Rajaram et al., "Experimental validation of the effects of microvasculature pigment packaging on in vivo diffuse reflectance spectroscopy," Lasers Surg. Med. 42(7), 680-688 (2010).

30. N. Rajaram et al., "Design and validation of a clinical instrument for spectral diagnosis of cutaneous malignancy," Appl. Opt. 49(2), 142-152 (2010).

31. A. D. Krainov et al., "Optical properties of mouse biotissues and their optical phantoms," Opt. Spectrosc. 115(2), 193-200 (2013).

32. R. Marchesini et al., "Ex-vivo optical-properties of human colon tissue," Lasers Surg. Med. 15(4), 351-357 (1994).

33. R. Hennessy et al., "Effect of probe geometry and optical properties on the sampling depth for diffuse reflectance spectroscopy," J. Biomed. Opt. 19(10), 107002 (2014). 
Mundo et al.: Diffuse reflectance spectroscopy to monitor murine colorectal tumor progression...

34. C. Neufert, C. Becker, and M. F. Neurath, "An inducible mouse model of colon carcinogenesis for the analysis of sporadic and inflammation-driven tumor progression," Nat. Protoc. 2(8), 1998-2004 (2007).

35. R. G. Azrak et al., "Therapeutic synergy between irinotecan and 5-fluorouracil against human tumor xenografts," Clin. Cancer Res. 10(3), 1121-1129 (2004).

36. A. Fioravanti et al., "Metronomic 5-fluorouracil, oxaliplatin and irinotecan in colorectal cancer," Eur. J. Pharmacol. 619(1-3), 8-14 (2009).

37. S. Orecchioni et al., "Vinorelbine, cyclophosphamide and 5-FU effects on the circulating and intratumoural landscape of immune cells improve anti-PD-L1 efficacy in preclinical models of breast cancer and lymphoma," Br. J. Cancer 118(10), 1329-1336 (2018).

38. L. Mielke et al., "Confocal laser endomicroscopy to monitor the colonic mucosa of mice," J. Immunol. Methods 421, 81-88 (2015).

39. D. Bettenworth et al., "Endoscopy-guided orthotopic implantation of colorectal cancer cells results in metastatic colorectal cancer in mice," Clin. Exp. Metastasis 33(6), 551-562 (2016).

40. E. Park, M. Cho, and C. S. Ki, "Correct use of repeated measures analysis of variance," Korean J. Lab. Med. 29(1), 1-9 (2009).

41. J. Pinheiro and D. Bates, Mixed-Effects Models in S and S-PLUS, Springer Science \& Business Media (2006).

42. R Core Team, R: A Language and Environment for Statistical Computing, Vienna (2018).

43. J. C. Pinheiro, B. Douglas, and S. DebRoy, "The R core team nlme: linear and nonlinear mixed effects models," in $R$ Package nlme Version, Vol. 3, pp. 1-83 (2007).

44. H. Wickham, ggplot2: Elegant Graphics for Data Analysis, Springer-Verlag, New York (2016).

45. T. M. Bydlon et al., "Chromophore based analyses of steady-state diffuse reflectance spectroscopy: current status and perspectives for clinical adoption," J. Biophotonics 8(1-2), 9-24 (2015).

46. B. S. Nichols, N. Rajaram, and J. W. Tunnell, "Performance of a lookup table-based approach for measuring tissue optical properties with diffuse optical spectroscopy," J. Biomed. Opt. 17(5), 057001 (2012).

47. K. Vishwanath et al., "Portable, fiber-based, diffuse reflection spectroscopy (DRS) systems for estimating tissue optical properties," Appl. Spectrosc. 65(2), 206-215 (2011).

48. R. Hennessy et al., "Monte Carlo lookup table-based inverse model for extracting optical properties from tissue-simulating phantoms using diffuse reflectance spectroscopy," J. Biomed. Opt. 18(3), 037003 (2013).

49. Y. Zhu et al., "Error removal by orthogonal subtraction (EROS): a customised pre-treatment for spectroscopic data," J. Chemom. 22(1-2), 130-134 (2008).

50. R. Reif et al., "Analysis of changes in reflectance measurements on biological tissues subjected to different probe pressures," J. Biomed. Opt. 13(1), 010502 (2008).

51. W. L. Chen et al., "Influence of contact state on NIR diffuse reflectance spectroscopy in vivo," J. Phys. D-Appl. Phys. 38(15), 2691-2695 (2005).

52. B. Cugmas et al., "Impact of contact pressure-induced spectral changes on soft-tissue classification in diffuse reflectance spectroscopy: problems and solutions," J. Biomed. Opt. 19(3), 37002 (2014).

53. N. Subhash et al., "Oral cancer detection using diffuse reflectance spectral ratio R540/R575 of oxygenated hemoglobin bands," J. Biomed. Opt. 11(1), 014018 (2006).

54. R. Mallia et al., "Oxygenated hemoglobin diffuse reflectance ratio for in vivo detection of oral pre-cancer," J. Biomed. Opt. 13(4), 041306 (2008).

55. A. P. Popov, A. V. Bykov, and I. V. Meglinski, "Influence of probe pressure on diffuse reflectance spectra of human skin measured in vivo," J. Biomed. Opt. 22(11), 110504 (2017).

56. Y. L. Ti and W. C. Lin, "Effects of probe contact pressure on in vivo optical spectroscopy," Opt. Express 16(6), 4250-4262 (2008).

57. M. F. Adam, M. J. Dorie, and J. M. Brown, "Oxygen tension measurements of tumors growing in mice," Int. J. Radiat. Oncol. Biol. Phys. 45(1), 171-180 (1999). 
58. J. C. Forster et al., "A review of the development of tumor vasculature and its effects on the tumor microenvironment," Hypoxia 5, 21-32 (2017).

59. G. J. Cerniglia et al., "Epidermal growth factor receptor Inhibition modulates the microenvironment by vascular normalization to improve chemotherapy and radiotherapy efficacy," PLoS One 4(8), e6539 (2009).

60. J. T. Liang et al., "Oncologic results of laparoscopic versus conventional open surgery for stage II or III left-sided colon cancers: a randomized controlled trial," Ann. Surg. Oncol. 14(1), 109-117 (2007).

61. Q. Pan et al., "Genomic variants in mouse model induced by azoxymethane and dextran sodium sulfate improperly mimic human colorectal cancer," Sci. Rep. 7(1), 25 (2017).

Ariel I. Mundo is a former Fulbright fellow and $\mathrm{PhD}$ student in the Biomedical Engineering Department at the University of Arkansas, Fayetteville. His research is focused on colorectal cancer, specifically on quantifying response to chemotherapy in the tumor and its microenvironment in vivo and in situ using optical techniques and genomics.

Gage J. Greening graduated with his $\mathrm{PhD}$ in biomedical engineering from the University of Arkansas in 2019 where he used diffuse reflectance spectroscopy (DRS) to quantify in-vivo tissue optical properties of subcutaneous murine colon carcinoma.

Michael J. Fahr, Jr.: Biography is not available.

Lawrence N. Hale is currently a biochemistry/pre-med junior at the University of Arkansas. His interests include skin diseases, radiology, and the development of different forms of cancer.

Elizabeth A. Bullard is an undergraduate student pursuing a Bachelor of Science degree in biomedical engineering at the University of Arkansas. Her research is focused on predicting mucosal healing in murine models of ulcerative colitis utilizing DRS and fluorescence lifetime imaging microscopy.

Narasimhan Rajaram is an assistant professor of biomedical engineering at the University of Arkansas. He received his $\mathrm{PhD}$ in biomedical engineering from the University of Texas at Austin and completed postdoctoral training at Duke University. His research is focused on the development of functional and molecular optical imaging strategies that can survey the tumor microenvironment and determine early response to therapy as well as long-term clinical outcome in terms of tumor recurrence and metastasis.

Timothy J. Muldoon received his $\mathrm{PhD}$ from Rice University in 2009 and his MD from the Baylor College of Medicine in 2010, and currently he is an associate professor of biomedical engineering at the University of Arkansas. 